\title{
Audiologic-Acoustic Evaluation of Speech Perception in Children with Cochlear Implants
}

\author{
O. Stieler ${ }^{a, b, *}$ A. SekUla ${ }^{c}$ And M. KARliK ${ }^{c}$ \\ ${ }^{a}$ Institute of Acoustics, Adam Mickiewicz University, Umultowska 85, 61-614 Poznań, Poland \\ ${ }^{b}$ Department of Otolaryngology, Poznań University of Medical Sciences \\ Przybyszewskiego 49, 60-355 Poznań, Poland \\ ${ }^{c}$ Department of Phoniatrics and Audiology, Poznań University of Medical Sciences, Poznań, Poland
}

\begin{abstract}
An early diagnosis of the congenital disorder of hearing creates new challenges for a multidisciplinary team: paedoaudiologists, ear nose throat specialists, and speech therapists. The cross modality matching method is based on the objective and subjective techniques in the evaluation of hearing thresholds in children. The electrical response audiometry provides information about the response of the brainstem to acoustic stimulation; the behavioural audiometry gives information about the perception and central associative processes in the auditory pathway. The paediatric fitting procedure relies on solid foundations of behavioural measurement to ensure the validity of hearing aid and cochlear implant fitting. This study assessed perception of phonemes in children with the cochlear implants and possibilities of applying acoustic solutions to the audiologic evaluation. The authors have also examined the possibilities of applying digital audio processing algorithm in clinical practice. Self-developed computer controlled diagnostic stations were used and tested. Speech perception was assessed on the basis of Erber's categories. Detection, discrimination and identification tests of 5 Ling phoneme were used. The sample comprised 23 implanted children, aged 3-6 years, who received a cochlear implant when they were 18 to 30 months old. The detection thresholds, discrimination and identification scores were assessed. Tests based on phonemes $a a, u u, i i, s s$, and $s h$ (5 sounds of Ling) were used. The results indicated significant correlations between pure tone audiometry results and the thresholds of phoneme detection [dB SPL]. The identification score in this group was $95-100 \%$.
\end{abstract}

PACS: 43.66. $+\mathrm{y}, 43.71 .+\mathrm{m}, 43.72 .+\mathrm{q}$

\section{Introduction}

A small set of words that would provide for the appropriate acoustic, energetic, phonematic and grammatical balance between tests and interaction during the tests are the basic problems with speech signal based tests involving children. Computer controlled tests eliminate the observation factor, which is extremely important in testing children with hearing impairment. Patients with hearing disorder very often compensate information deficiency using sense of vision. It is very important in children to exclude during testing observations and reactions based on visual stimuli. In case of software controlled behavioral test it is not necessary to deflect attention of the child by an assistant.

Application of described signals enables standardization of the test in speech therapists room. Implanted patients live in different locations, very often far away from each other. So unification of test conditions is necessary which are performed by acousticians, audiologists and speech therapists in cochlear implant center and local outpatient wards.

\footnotetext{
* corresponding author; e-mail: osaku@umed.poznan.pl
}

IT tools based on digital signal processing methods permit any configuration and editing of the sound materials used as a stimulus in the study and precise adaptation of the program to meet preferences of individual specialists who evaluate the child's hearing (audiologist, speech therapist, hearing aid consultant and acoustician) [1]. The evaluation of the usefulness of the tests is based on the methods of objective diagnostics of the auditory pathway, well known and used in clinical practice, such as measurement of otoacoustic emission, stappedius reflex threshold and evoked auditory brainstem response $[2,3]$. Electrically evoked neural response telemetry is another tool used in objective evaluation in the case of children with significant hearing impairment who have cochlear implants $[4,5]$.

\section{Material}

The study comprised 24 children who had a cochlear implant fitted at the age of 18-30 months, presently 3-6 years old, without any other disorders. Speech perception was assessed 3 years after implantation. Studies in the group described above include patients implanted at the age critical for the correct development of speech. The evaluation of the development of hearing skills was based on the Erber classification, natural for the development 
of hearing skills. The evaluation comprised detection, discrimination, and identification of such speech elements as phonemes, syllables and words.

\section{Methods}

The experiment comprised an early assessment of the perception of speech elements, conforming to the development of the hearing skills of children who were implanted at an early age [6]. The authors used their own acoustic and IT solutions. The audiologic-acoustic assessment of speech perception was made in line with the development level of the communication process, individually determined for each patient.

Speech development periods:

melody period $(0-1$ years $)$ :

- vocal forms preceding articulated speech: screaming, cooing, babbling, echolalia,

- end of preparatory period: fully ready to imitate the speech of adults,

word period (1-2 years):

- acquisition of new object-language associations thanks to the confrontation with the speech of the surroundings and correction by parents,

- active use of one and two-word utterances,

sentence period (2-3 years):

- speech is further perfected,

- use of 2-3 word sentences (declarative, imperative and interrogative sentences),

- perfection of phonematic hearing.

Patients were selected based on multidisciplinary team, consisting of audiologist, acoustician, educator and speech therapists. For evaluation of auditory skills during discrimination and identification process, only children with no development disturbances during rehabilitation and auditory-verbal communication, appropriate to patient's age and time of cochlear implant using were chosen. Test of 5 Ling sounds consists of phonems with different spectral characteristics [7]. It enables estimation of detection, discrimination and identification thresholds, so important for speech perception in the frequency domain. Due to using the most simple speech elements in closed set, it was possible to evaluate phonems discrimination and identification score in the small patients from the study group [7]. Children had got used to the tested signals during systematically done rehabilitation sessions. The experimenters used elements of the speech signal since it was possible to evaluate central processes that take place in the brain during such complex activities of hearing perception as discrimination and identification [8]. This is the basic criterion used to evaluate whether adjustment of the speech processor will be successful, i.e. whether the communicative process will be restored. The method described here also allows for the estimation of the most comfortable level (MCL), while the pure tone audiometry (PTA) only allows for the assessment of the potential with which information will be delivered within the hearing threshold.

In view of the acoustic characteristic of the signal, speech sounds are complex non-harmonic signals. They can be compared to noise bands (fricatives and affricates), acoustic impulses (plosives) and multi-tones (vowels). In the spectral analysis in consonant spectra spectral maxima, i.e. formants, can be identified, which differentiate consonants with respect to frequency. A classical spectral analysis is based on a two-dimensional characteristic of the signal - the $X$-axis - frequency and the $Y$-axis - amplitude. The spectral characteristic of 5 Linga phonemes, used in the tests, is presented in Fig. 1.

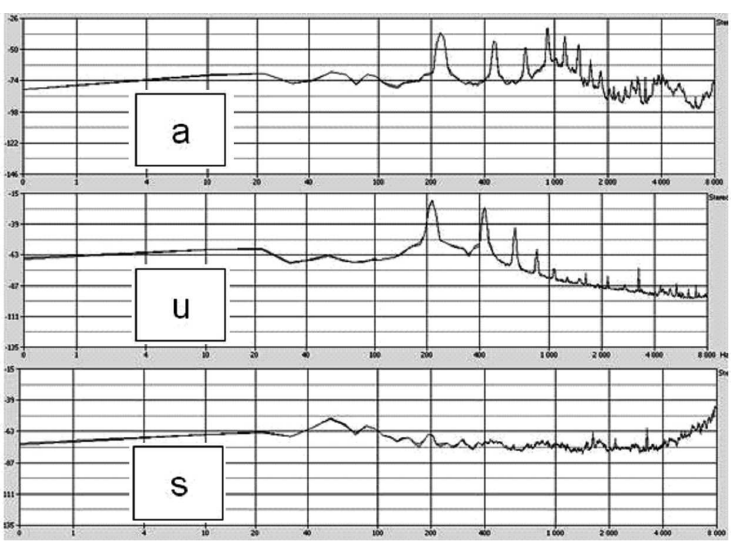

Fig. 1. Examples of spectral characteristic of Linga phonemes for sounds $a, u, s$.

The study comprised a digital recording, acoustic analysis and processing of the sound material. The voice of the professional speech therapist was recorded in the SoundForge program, using the computer controlled external EMU 1616 sound card and Sennheiser microphone. The levels of each sample were calibrated in a free acoustic field [9] against the calibration signal, i.e. BabbleNoise, based on the word units of the Polish Frequency Dictionary. Calibration was performed using the Svantec Svan 945 sonometre in an anechoic chamber in which the study was conducted. The level of the acoustic background measured was as recommended in the standard.

Context dedicated software of the measurement station was prepared. Depending on the profession of the therapist who logged into the database and who conducted the test, different descriptions of the signals used could be entered into the program. A clinical engineer could access results of fast Fourier transform (FFT) based signal analysis, an audiologist - a description based on the audiogram of the hearing field, and a speech therapist the results of the spectrograms (Fig. 2). 
Audiometry in free field [9] was done with active speech processor with program number, volume setting and microphone sensitivity setting actually used by the patient. Test was performed $1.5 \mathrm{~m}$ from the speaker in anechoic chamber, for frequencies used in pure tone audiometry: $0.25,0.5,1,2,3,4,6 \mathrm{kHz}$. During pre-test demonstration signals were presented using decreasing method, from the level individually set for hearing threshold: $40-50 \mathrm{~dB}$ SPL and $10-20 \mathrm{~dB}$ SL.
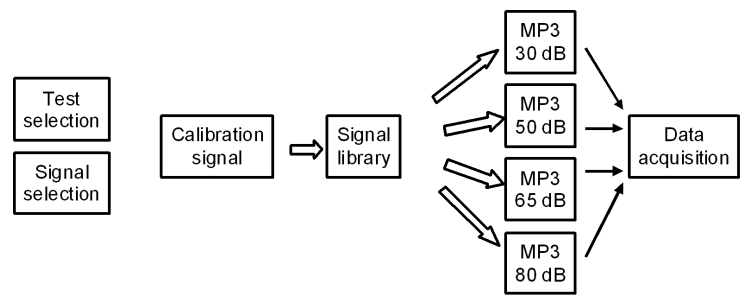

Fig. 2. Logical structure of the program.

\section{Results}

\subsection{Discrimination tests}

Based on the acoustic analysis of the speech signal phonemes were divided into two groups - low and high frequency phonemes. The group of low frequency phonemes comprised $a h$, oo, and ee phonemes. The group of high frequency phonemes comprised $s s$, sh phonemes. Discrimination was assessed for the phonemes in each group.

In the case of phonemes with a large spectral contrast, originating from different groups, the subject's task was easier than in the case of phonemes in the same group. The study was based on the $2 \mathrm{AAFC}$ procedure. The child tested was asked to say whether the phonemes compared were the same or different. Before the child could answer the question, the speech therapist had to prepare the child for the test through systematic hearing training (Fig. 3). Tests after the calibration of the free field system were made for the signal acoustic pressure levels of 50,65 and $80 \mathrm{~dB}$ PL. The values of the identification coefficient [\%] measured for each patient are given in Fig. 4.

Otoacousic emissions were recorded in all the patients and result: REFER was noticed in all frequency bands from 1 to $6 \mathrm{kHz}$, repeated at least in 3 independent tests.

Impedance audiometry: during tympanometry in both ears type $A$ curve was registered, and no stapedius reflex was noticed during stimulation at $100 \mathrm{~dB}$ for frequency $0.5,1,2,4 \mathrm{kHz}$ at implanted ear and $90-100 \mathrm{~dB}<1 \mathrm{kHz}$ in contralateral to CI, acoustic aided ear [1]. Neural response telemetry (NRT) [10] was recorded in all the patients and the NRT thresholds were found in all the patients during stimulation over $70 \%$ of active electrodes in the range between $\mathrm{T}$ (threshold) and $\mathrm{C}$ (comfort) electrical stimulation level.

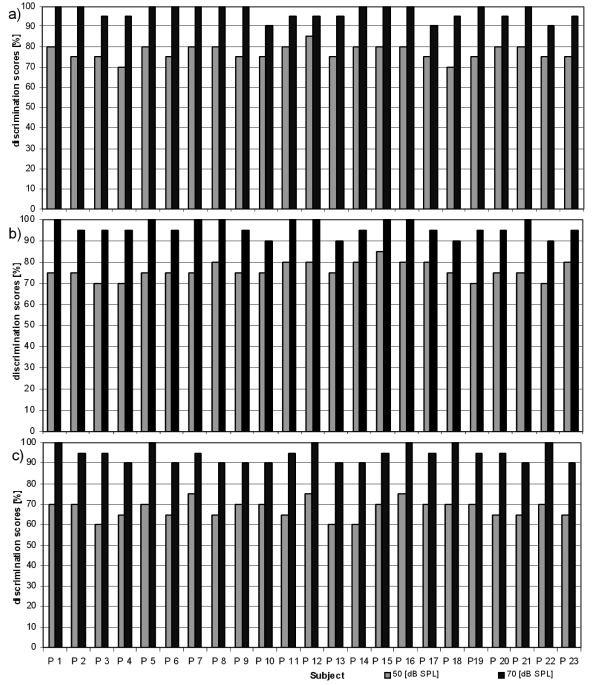

Fig. 3. Discrimination scores: (a) $a h, o o, e e-s s, s h$; (b) $a h, o o, e e-o o, e e, a h$; (c) $s s-s h$.

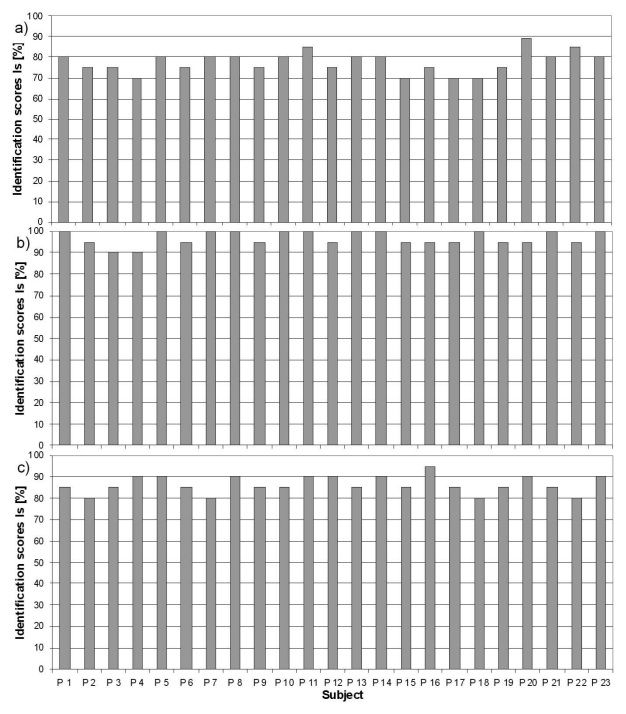

Fig. 4. Identification scores: (a) $50 \mathrm{~dB}$ SPL, (b) $65 \mathrm{~dB}$ SPL, (c) $80 \mathrm{~dB}$ SPL.

\section{Discussion}

The tested patients had difficulties with differentiation of phonemes based on high-frequency characteristics $(s s-s h)$. It is confirmed by long-term observations made by speech therapists during rehabilitation process. The most difficult task is discrimination and identification of these phonemes even having normal tone signal detection thresholds in free field (20-30 dB SPL; $0.25-6 \mathrm{kHz})$. Polish language, similar to other Slavic languages, consists of large number of phonemes with high frequency sounds. So it is necessary to pay attention to program gain, T-level, number of maxima and manual change of frequency allocation table (FAT) during speech processor 
fitting. Influence of these parameters on discrimination and identification results in larger group of tested children is the next stage of the research.

Estimation of MCL concludes directly from the level when the patient reaches maximal value of identification score. For the group of tested patients optimal level was set to $65 \mathrm{~dB}$ SPL, which is appropriate for normal level of voice production. An interesting thing could be that identification score is worse for level of $80 \mathrm{~dB}$. It could be explained as a result of compression system during preprocessing of signal in the speech processor.

\section{Conclusions}

The project helped to develop and assess the clinical results of the diagnostic tool, which can be used to make an early evaluation of the perception of speech elements. Based on the verification of the electrophysiological analyses (ABR, EABR, NRT), benefits for patients with cochlear implants were evaluated. Application of a multi-specialist software platform helped to evaluate the hearing threshold (detection), discrimination and identification of speech signal elements.

- The method permits the evaluation of perception based on tests of detection, discrimination and identification;

- The solutions permit full control over the stimulus signal;

- The results confirm the usefulness of the digital processing of sounds in clinical practice;

- In the long term assessment, the identification coefficient in the group studied was $95-100 \%$.

\section{Acknowledgments}

O.S. acknowledges the financial support extended by the Polish Ministry of Sciences and Education: grant No. N 504038 32/3138.

\section{References}

[1] O. Stieler, D. Komar, A. Sekula, Bull. Am. Auditory Society 33, 52 (2008).

[2] O. Stieler, A. Sekula, M. Karlik, in: Some Aspects of Medicala Physics - In Vivo and In Vitro Studies, Vol. 1, Ed. Z. Drzazga, University of Silesia, Katowice 2010, p. 46.

[3] O. Stieler, A. Sekula, D. Komar, Bull. Am. Auditory Society 33, 51 (2008).

[4] G.F. Smoorenburg, C. Willeboer, J.E. Van Dijk, Audiol. Neurootol. 7, 6 (2002).

[5] D.A. Nelson, J.L. Schmitz, G.S. Donaldson, N.F. Viemeister, J. Acoust. Soc. Am. 100, 4 (1996).

[6] N. Erber, Auditory Training, Alexander Graham Bell Association for the Deaf, Washington DC 1982.

[7] D. Ling, Foundations of Spoken Language for the Hearing-Impaired Child, Alexander Graham Bell Association for the Deaf, Washington DC 1989.

[8] O. Stieler, A. Sekula, Pol. J. Med. Phys. Eng. 15, 901 (2009).

[9] ISO 8253, Acoustics-Audiometric Test Methods. Sound field audiometry with pure tone and narrow-band test signals, International Organization for Standardization, Geneva 1996.

[10] T. Morita, Y. Naito, T. Hirai, S. Yamaguchi, J. Ito, Eur. Arch. Oto-Rhino-Laryngol. 260, 2 (2003). 